\title{
Rotavirus-mediated alteration of gut microbiota and its correlation with physiological characteristics in neonatal calves
}

\author{
Ja-Young Jang $^{1 \dagger}$, Suhee Kim ${ }^{2 \dagger}$, Min-Sung Kwon ${ }^{1}$, \\ Jieun Lee ${ }^{1}$, Do-Hyeon $\mathrm{Yu}^{3}$, Ru-Hui Song ${ }^{4}$, \\ Hak-Jong Choi ${ }^{1 \star}$, and Jinho Park ${ }^{4 \star}$ \\ ${ }^{1}$ Research and Development Division, World Institute of Kimchi, \\ Gwangju 61755, Republic of Korea \\ ${ }^{2}$ Division of Animal Diseases and Health, National Institute of Animal \\ Science, Rural Development Administration, Wanju 55365, Republic of \\ Korea \\ ${ }^{3}$ Institute of Animal Medicine, College of Veterinary Medicine, \\ Gyeongsang National University, Jinju 52828, Republic of Korea \\ ${ }^{4}$ Department of Veterinary Internal Medicine, College of Veterinary \\ Medicine, Chonbuk National University, Iksan 54596, Republic of Korea \\ (Received Oct 1, 2018 / Revised Oct 31, 2018 / Accepted Nov 1, 2018)
}

Diarrhea is a fatal disease to neonatal calves, and rotavirus is the main pathogen associated with neonatal calf diarrhea. Although previous studies have reported that the gut microbiota is changed in calves during diarrhea, less is known about whether rotavirus infection alters the structure of the gut microbiota. Here, we characterized fecal microbial communities and identified possible relationships between the gut microbiota profiles and physiological parameters. Five fecal specimens of rotavirus-infected calves from 1 to 30 days after birth and five fecal specimens of age-matched healthy calves were used for the microbial community analysis using the Illumina MiSeq sequencer. Rotavirus infection was associated with reduced rotavirus infection significantly reduced the richness and diversity of the bacterial community. Weighted unique fraction metric analysis exhibited significant differences in community membership and structure between healthy and rotavirus-infected calves. Based on relative abundance analysis and linear discriminant analysis effect size, we found that the representative genera from Lactobacillus, Subdoligranulum, Blautia, and Bacteroides were closely related to healthy calves, while the genera Escherichia and Clostridium were closely affiliated to rotavirus-infected calves. Furthermore, canonical correlation analysis and Pearson correlation coefficient results revealed that the increased relative abundances of Lactobacillus, Subdoligranulum, and Bacteroides were correlated with normal levels of physiological characteristics such as white blood cells, blood urea nitrogen, serum amyloid protein $A$, and glucose concentration in serum. These results suggest that rotavirus infection alters the structure of the gut microbiota, correlating changes in physiological

\footnotetext{
${ }^{\dagger}$ These authors contributed equally to this work.

*For correspondence. (J. Park) E-mail: jpark@jbnu.ac.kr; Tel.: +82-63-8500949; Fax: +82-63-850-0910 / (H.J. Choi) E-mail: hjchoi@wikim.re.kr; Tel.: +82-62-610-1729; Fax: +82-62-610-1850

Copyright (c) 2019, The Microbiological Society of Korea
}

parameters. This study provides new information on the relationship between gut microbiota and the physiological parameters of rotavirus-mediated diarrheic calves.

Keywords: diarrhea, rotavirus, gut microbiota, physiological parameters

\section{Introduction}

Neonatal calf diarrhea (NCD) is a common cause of growth disorder and death in newborn calves and leads to economic loss in livestock farms (Cho and Yoon, 2014). A high mortality risk for NCD has been reported in calves that are less than one month old (Uetake, 2013). According to the 2007 National Animal Health Monitoring System (NAHMS) for the U.S. dairy, diarrhea accounted for $57 \%$ of total deaths in weaning calves (2008) and a similar mortality rate (53.4\%) resulting from calf diarrhea has recently been reported in Korea (Hur et al., 2013).

Calf diarrhea is a complex and multifactorial disease that can be triggered by various factors (Cho and Yoon, 2014). Numerous infectious pathogens are involved in calf diarrhea. Rotavirus, coronavirus, Escherichia coli, and Cryptosporidium species are regarded as the most important NCD-causing enteropathogens (Gulliksen et al., 2009). Notably, rotavirus is known to be one of the frequently identified enteropathogens in feces from young calves (Izzo et al., 2011). It is orally infected to calves, replicates in the cytoplasm of intestinal epithelial cells, and destroys mature intestinal cells (Cho and Yoon, 2014). It is a highly contagious viral pathogen that causes severe watery diarrhea in young calves within 2 weeks and a strong pathogenic agent that can lead to death due to dehydration by severe diarrhea in newborn calves within 1 week (Kapikian and Chanock, 1996; Ammar et al., 2014).

Infection by enteropathogens causes an alteration of normal gastrointestinal microbiota, which is thought to exacerbate diarrhea (Rolhion and Chassaing, 2016; Wotzka et al., 2017). Disruption of gastrointestinal microbiota may lead to the promotion of intestinal inflammation, modulation of immune response, and loss of ability to compete with pathogens for nutrients, which are subsequently thought to be closely related to changes in the physiological components of the calves (Rolhion and Chassaing, 2016; Gomez et al., 2017). In addition, the oral administration of antibiotics and medicines can significantly change gastric $\mathrm{pH}$ and are expected to adversely affect the normal bacterial flora, yeast, and protozoa of the stomach and intestines (Penders et al., 2006). For the treatment and management of gastrointestinal diseases in calves, it is necessary to understand the mechanisms 
of changes in intestinal microbiota and to analyze the correlation with the changes of physiological factors according to the causes of diseases. However, little is known about gut dysbiosis and the enteropathogens involved in NCD, and their-related physiological changes.

In the study, we compared the distribution of intestinal microbiota among normal calves and calves infected with rotavirus by next-generation sequencing of the 16S rRNA gene. In addition, we identified possible correlations between certain microbiota and physiological parameters. Our aim is to offer strategies to prevent NCD via rotavirus-related gut-microbiota and physiological changes.

\section{Materials and Methods}

\section{Animals and sampling}

Calves under 30 days of age were randomly selected from seven farms in the Republic of Korea from 2016 to 2017. All fecal samples obtained from calves were examined for the pathogens detection, such as coronavirus, rotavirus, Cryptosporidium spp., E. coli $\mathrm{K} 99+$, and a giardia using a rapid test kit (Bio-note) and for the Eimeria species detection performing fecal parasite test (floating/sedimentation test). For fecal parasite test, fecal material is mixed with a special liquid (ex, sugar solution) that causes the parasite eggs to float to the surface. The eggs are collected from the surface and observed the appearance to identify parasite type using microscope. In addition, fecal samples that were positive for rotavirus were confirmed by real time PCR, as previously reported (Cho et al., 2010). Based on the above criteria, the experimental group was divided into two as follows; five normal fecal samples (17-30 days of age, 2 females and 3 males) without diarrhea and any pathogens (healthy group) and five fecal samples (3-27 days of age, 2 females and 3 males) positive for only rotavirus (rota group). Fecal samples were stored at $-80^{\circ} \mathrm{C}$ until processing gut microbiota analysis. All procedures were carried out according to ethical guidelines for the use of animal samples, as approved by Chonbuk National University (Institutional Animal Care and Use Committee [IACUC] Decision No. CBU 2014-00026).

\section{Evaluation of physiological components}

Blood work was carried out on the calves: the complete blood count (CBC) included a white blood cell (WBC) count consisting of 5 differentials and the red blood cell (RBC) and platelet indices by a Procyte Dx hematology analyzer (IDEXX Laboratories), and the serum biochemistry tests were used to analyze 8 parameters (blood urea nitrogen [BUN], glucose, $\mathrm{Na}, \mathrm{K}, \mathrm{Cl}$, phosphate, total protein, and albumin) using a Catalyst One ${ }^{\mathrm{TM}}$ chemistry analyzer (IDEXX Laboratories). In order to evaluate systemic inflammation, serum acute phase proteins such as serum amyloid A (SAA) and haptoglobin concentration were also determined by sandwich ELISA (DuoSet ELISA Development kit, R\&D Systems). Fibrinogen was evaluated using Millar's method (Millar et al., 1971).

\section{DNA extraction, amplification, and sequencing of bacterial 16S rRNA gene}

For the bacterial gene sequence, total DNA was extracted from $200 \mathrm{mg}$ (wet weight) of fecal samples using a FastDNA Spin Kit (MP Biomedicals). The V3-V4 region of the $16 \mathrm{~S}$ rRNA gene was amplified with $341 \mathrm{~F}$ primer and 805R primer. Then, secondary amplification for attaching the Illumina NexTera barcode was performed with i5 forward primer and 7 reverse primer. The condition of secondary amplification is equal to the former except that the amplification cycle was set to 8 . The amplified products were purified using a QIAquick PCR purification kit (Qiagen). Equal concentrations of purified products were pooled together and short fragments were removed (non-target products) using Ampure beads kit (Agencourt Bioscience). The quality and product size were assessed on a Bioanalyzer 2100 (Agilent) using a DNA 7500 chip. Mixed amplicons were pooled and sequencing was carried out at Chunlab Inc., using the Illumina MiSeq Sequencing system (Illumina) according to the manufacturer's instructions.

\section{Microbiome analysis}

The QIIME software package was used for the bioinformatics analysis. Paired-end reads were merged to obtain fully overlapping reads which were then aligned to the Greengene reference database. Sequences that were misaligned with the target region were removed. Uchime was utilized to identify chimeras, which were then removed. Sequences belonging to non-bacterial domains (chloroplasts, mitochondria, archaea, and eukaryotes) also were removed. Samples were completed to normalize sequence number by randomly selecting several sequences that corresponded to the lowest number of reads for any sample. Unweighted unique fraction metric (UNIFRAC) analysis, analysis of molecular variance (AMOVA), homogeneity of molecular variance analysis (HOMOVA), and parsimony test were used to compare community membership and structure between groups. The graph of the microbial changes between the healthy and the rotavirus diarrheic calves was derived from a discriminant analysis using bacterial genus prevalence as covariates and disease status (healthy or rotavirus diarrhea) as the categorical variable by dendrogram and principal coordinate analysis (PCoA) plots. Linear discriminant analysis effect size (LEfSe) was used to identify bacterial taxa that were enriched in the feces of healthy and diarrheic calves, based on a $P<0.05$ and an LDA score $>2.0$. LEfSe was performed online using the Galaxy workflow framework (https://huttenhower.sph. harvard.edu/galaxy/).

\section{Statistical analysis}

The relative abundances of the main phyla, classes, orders, and families (median relative abundance $>0.1 \%$ ) and the main genera (median relative abundance $>0.01 \%$ ) were calculated. Comparison of the relative abundances between groups (healthy and rotavirus diarrhea) was performed using the nonparametric Mann-Whitney $U$-test via GraphPad Prism 6. Clustering of the groups was represented by principal coordinate analysis, canonical correlation analysis, and Pearson correlation coefficient plotted by statistical software using XLSTAT 2018. 


\section{Results}

\section{Clinical findings of calf diarrhea}

In the rotavirus-infected group, the leukocyte and neutrophil counts were significantly increased $[12.1 \pm 1.6 \mathrm{~K} / \mu \mathrm{l}$; $48.7 \pm 13.1 \%]$ compared with the control group $[9.1 \pm 2.3$ $\mathrm{K} / \mu \mathrm{l} ; 38.8 \pm 15.7 \%$ ] (Fig. 1). The BUN [12.6 $\pm 6.2 \mathrm{mg} / \mathrm{dl}]$ was significantly increased, and the glucose value [84.4 \pm $19.2 \mathrm{~g} / \mathrm{dl}]$ was decreased compared with the control group [9.8 $\pm 3.6 \mathrm{mg} / \mathrm{dl} ; 110.4 \pm 17.2 \mathrm{~g} / \mathrm{dl}]$ (Fig. 1). SAA concentration was also significantly elevated.

\section{Analysis of 16S rRNA gene sequencing}

DNA representing the intestinal microflora from 10 subjects, including 5 diarrheic calves infected with rotavirus, was isolated from feces, and the V3-V4 regions in the 16S rRNA gene pool were amplified and sequenced using MiSeq. We obtained a total of 575,028 reads and a mean of 57,502 reads per calf (standard deviation, 5,030; range, 52,800 to 66,835 ). This was a good coverage quality result of samples analyzed by the microbial diversity of the feces. Figure $2 \mathrm{~A}$ presents a rarefaction analysis showing the operational taxonomic unit (OTU) levels for healthy and diarrheic calves. The shape of the rarefaction curves indicates that the sequencing depth reached was the maximum in this study.

\section{Alpha-diversity}

The Simpson's, Shannon's, and Chao-1 indices were calculated to confirm evenness, diversity, and richness, respecti- vely. The Wilcoxon rank sum test was used to compare the groups. The richness, diversity, and evenness of gut microbiota between healthy and diarrheic calves were found to be significantly different (Fig. 2B). Chao-1 and Shannon indices significantly decreased in rotavirus-infected calves, whereas the Simpson index significantly increased in rotavirus-infected calves. The core bacteria were examined by identifying the genus with relative abundances of at least $0.1 \%$ in all samples. These results indicate that rotavirus infection alters the diversity, evenness, and richness of the gut microbiota.

\section{Community membership and structure}

The findings that rotavirus infection significantly affects alpha-diversity of the gut microbiota prompted us to examine the community membership and structure of gut bacteria. The dendrogram and PCoA scores plot illustrated a degree of clustering in relation to the diarrheic calves compared to healthy calves (Fig. 2C). In addition, PCoA plots revealed differences in gut microbiota composition among the two groups (Fig. 2D). Percentage values of the axes represented the contribution of the principal coordinate to the description of the total variance in the dataset. These results suggest that rotavirus infection alters the community membership and structure of the gut microbiota.

\section{Relative abundance and core microbiota}

In order to investigate the bacterial community structure showing significant differences, community membership between healthy and rotavirus-infected calves were compared
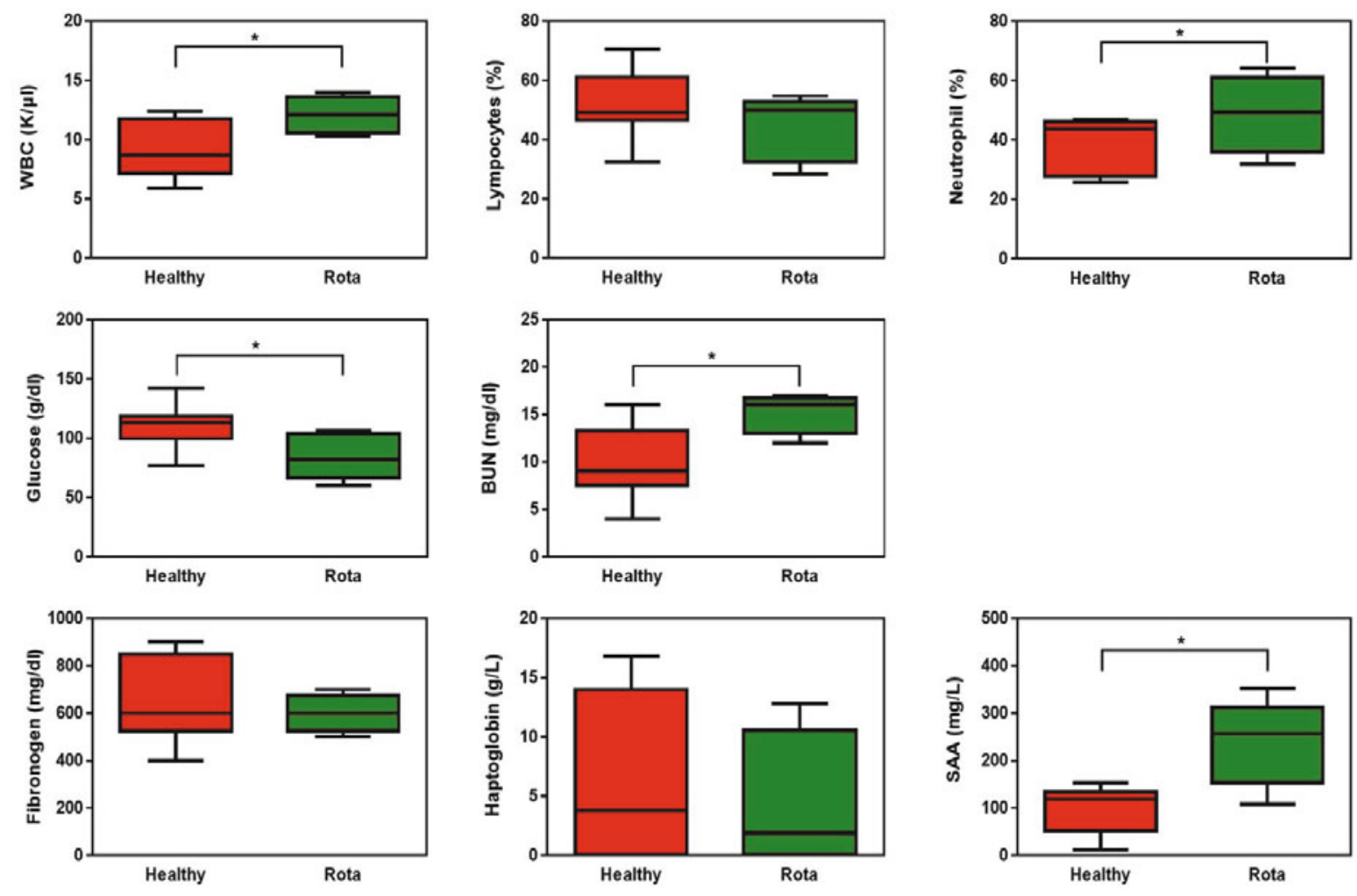

Fig. 1. Principal hematological and biochemical changes between the healthy and rotavirus-infected group. ${ }^{\star} P<0.05$. 
(A)

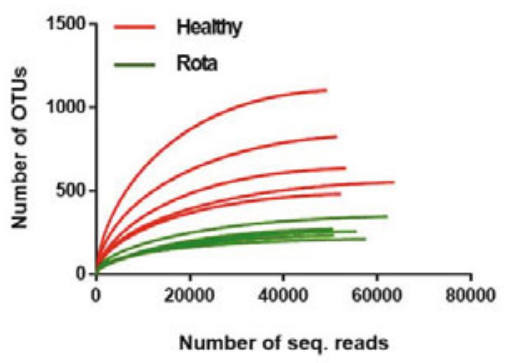

(C)

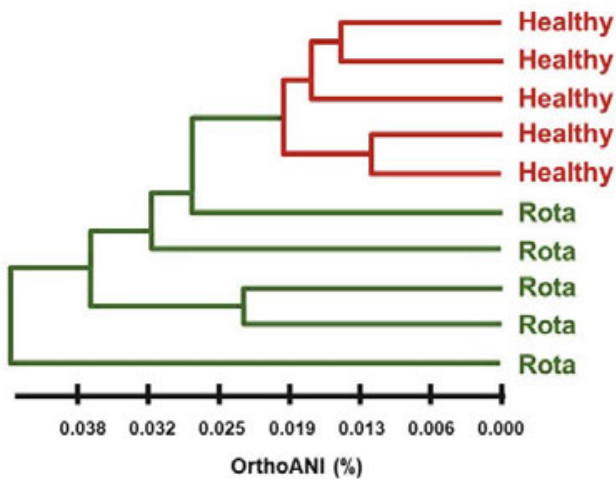

(B)
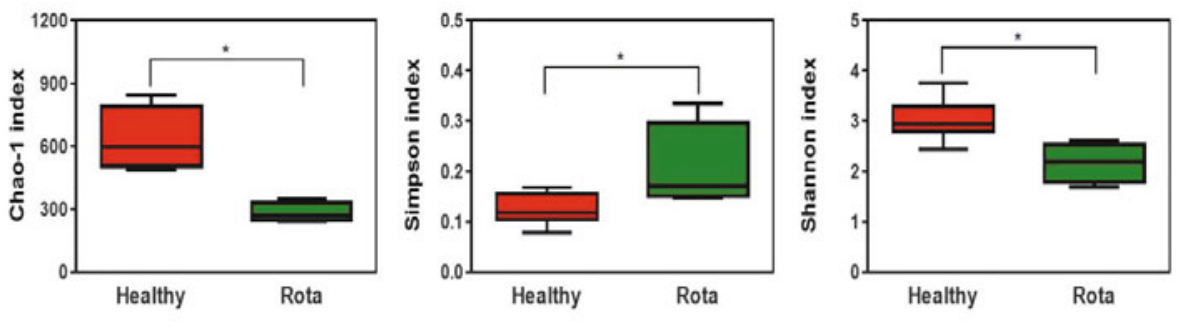

PC3 12.35\%

(D)

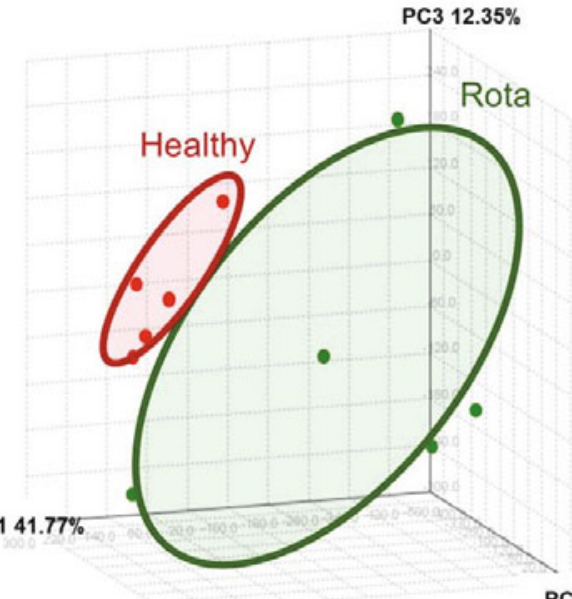

PC2 27.29\%

Fig. 2. Diversity profiles of the gut microbiota. (A) The rarefaction curve; (B) Box plots of alpha-diversity (internal diversity) in each microbiota comparing healthy and rotavirus diarrhea. Richness (Chao-1), diversity (Simpson), and evenness (Shannon) indexes observed in healthy and diarrheic calves; (C) Dendrogram of beta-diversity among samples; (D) Discriminant analysis of beta-diversity among samples. The microbial alteration between healthy (red) and rotavirus diarrhea (green) is illustrated by the line or circle, and individual data are also depicted. ${ }^{*} P<0.05$.

(A)

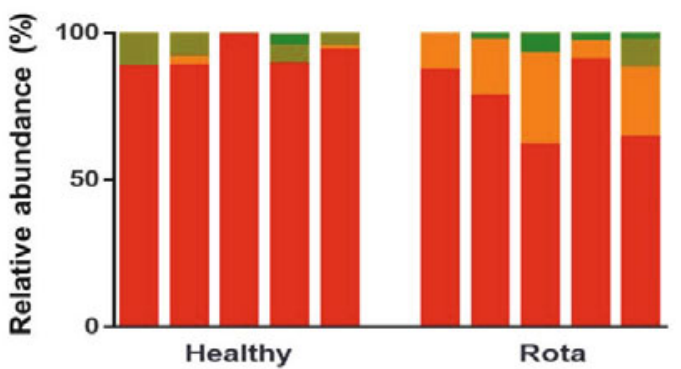

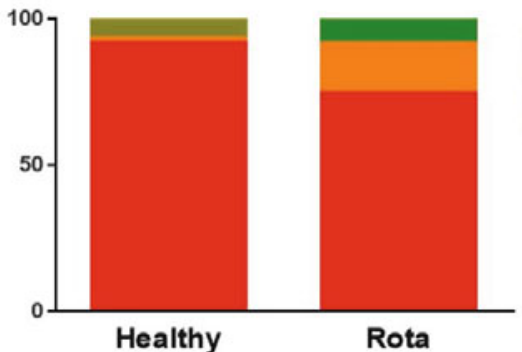

Actinobacteria

Bacteroidetes

Proteobacteria

Firmicutes
(B)

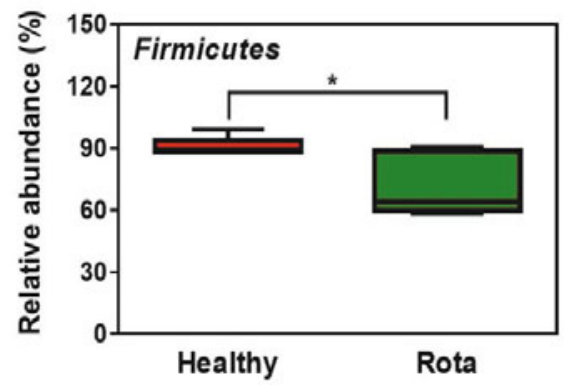

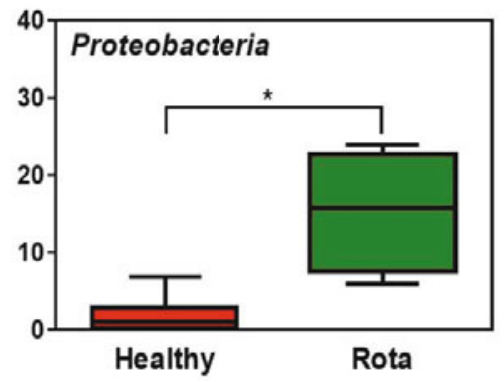

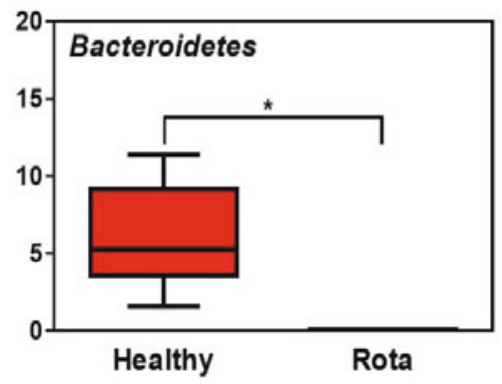

Fig. 3. Relative abundance of main bacterial phyla in the feces of healthy and rotavirus diarrhea calves. (A) Individual and mean relative abundance of the main bacterial phyla ( $>0.5 \%$ of total of sequences); (B) Bacterial phyla that are distinctive between the healthy and rotavirus diarrhea microbiota. ${ }^{\star} P<0.05$. 
from the phylum to the genus level. A total of twenty different phyla were identified, however, more than 98\% of Firmicutes, Proteobacteria, and Bacteroidetes were present in both healthy and rotavirus diarrheic calves (Fig. 3A). In healthy calves, Firmicutes $(P=0.0093)$ and Bacteroidetes $(P=0.0041)$ were relatively abundant, and Proteobacteria $(P=0.0014)$ were markedly less present than in rotavirus diarrheic calves (Fig. 3B). Fifty-five different classes, 112 orders, and 241 families were identified, but only 17,31 , and 46 accounted for $>0.1 \%$ of the total sequences, respectively. Overall, 596 genera were detected, which includes 166 genera with over $0.01 \%$ relative abundance of total sequences (data not shown).

\section{LEfSe analysis}

We conducted a LEfSe analysis to identify the top discrimi-

(A)
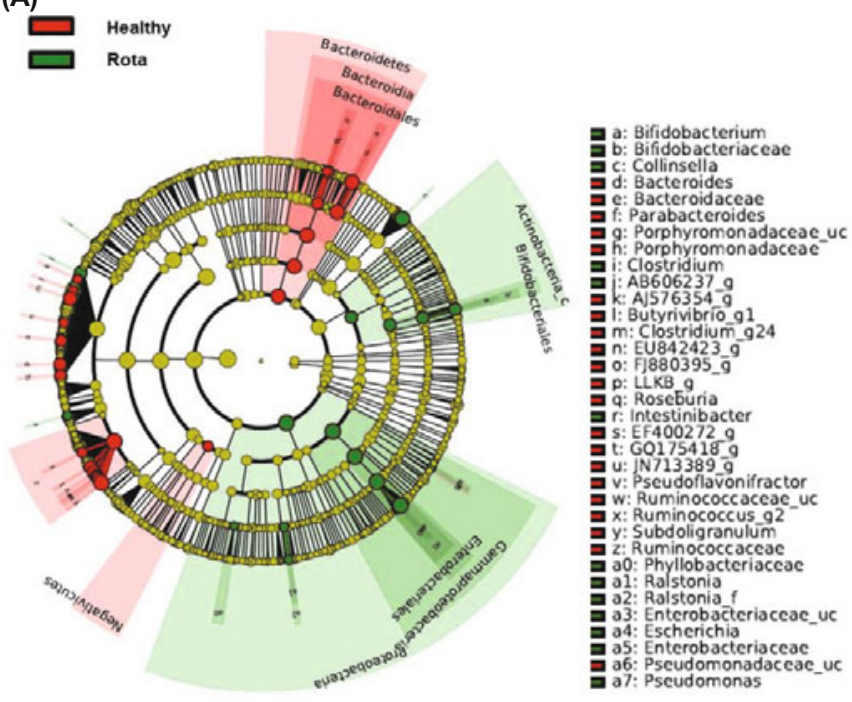

natory core microbiota at the genus level distinctive between the healthy and rotavirus-infected calves. In comparison between the healthy and rotavirus diarrheic calves, 17 and 10 genera were enriched in healthy and diarrhea feces, respectively (Fig. 4A). The rich phylotypes derived from healthy samples were mainly in the class Bacteria, whereas most of the rotavirus diarrhea samples consisted of Actinobacteria and Gammaproteobacteria (Fig. 4A). The 11 genera including Subdoligranulum, Bacteroides, and Clostridium_g24 were associated with healthy calves, whereas 8 genera including Escherichia, Collinsella, and Clostridium were significantly associated with rotavirus-infected calves (Fig. 4B). As shown in Fig. 4C, the relative abundances of Lactobacillus, Subdoligranulum, Blautia, and Coprococcus_g2 were reduced, while Escherichia, Clostridium_g21, Streptococcus, and Clostridium increased in rotavirus-infected calves.

(B)

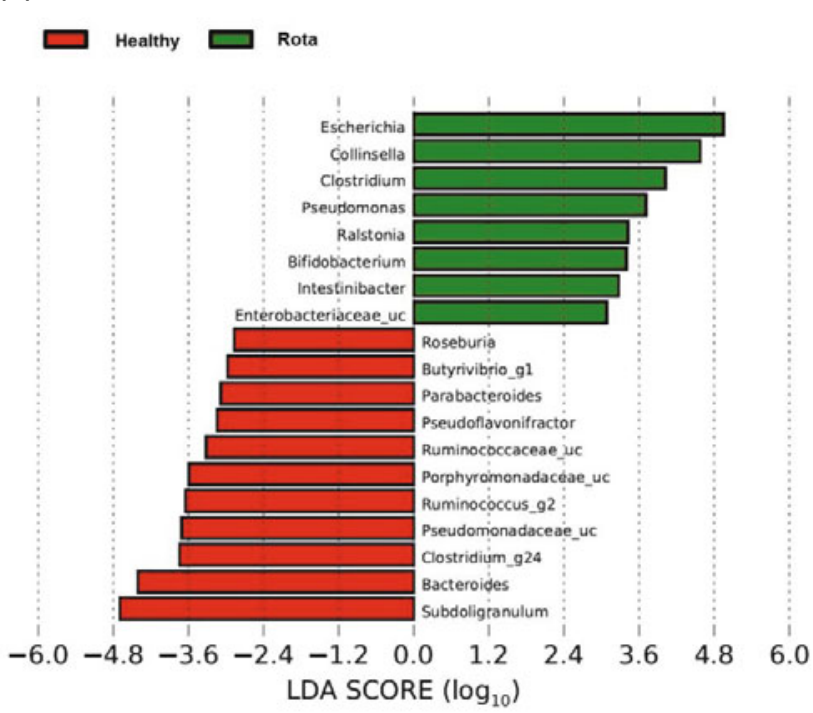

(C)
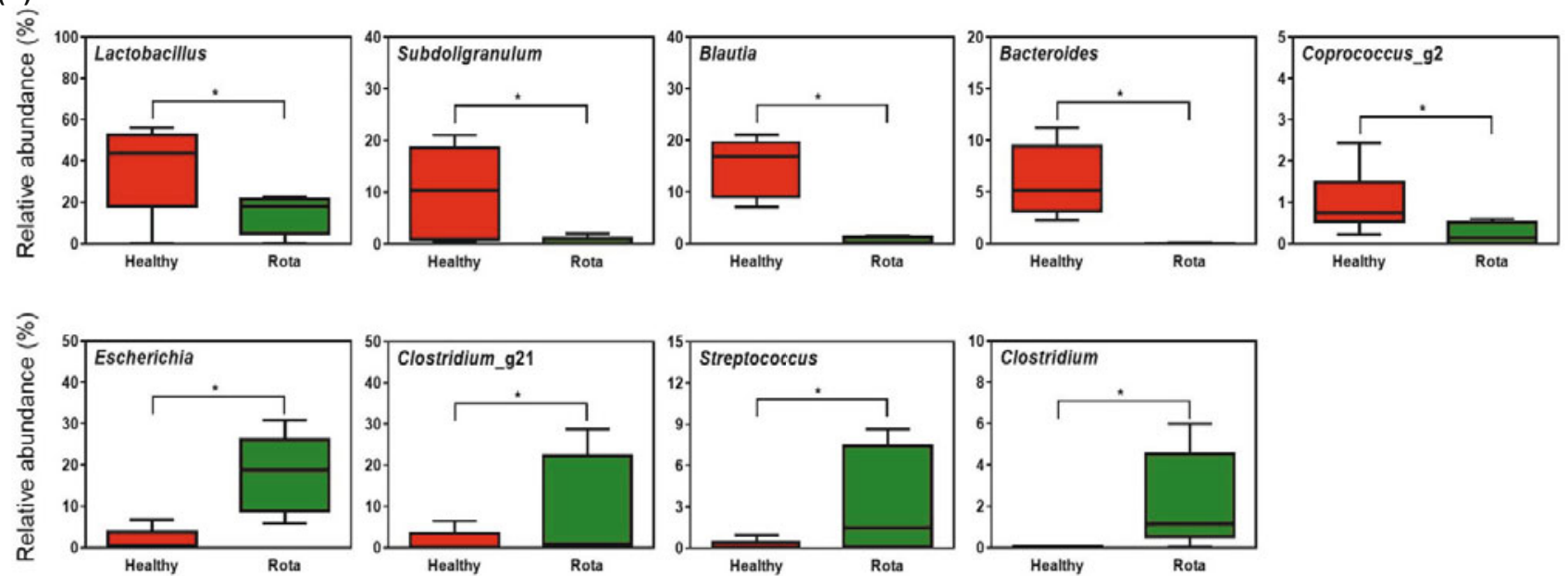

Fig. 4. Distinctive genera in the feces of healthy and rotavirus diarrhea calves. (A) Cladogram plotted from LEfSe analysis showing the taxonomic levels represented with phyla in the outermost the ring and genera in the innermost ring. Each circle is a member within that level; (B) Plot from LEfSe analysis indicating enriched genera associated either with healthy (red) or rotavirus diarrhea (green); (C) Relative abundance of the main bacterial genera in feces of healthy and rotavirus diarrhea by Mann-Whitney test. ${ }^{*} P<0.05$. 
(A)

\section{$\square$ Healthy \\ $\square$ Rota}

(B)

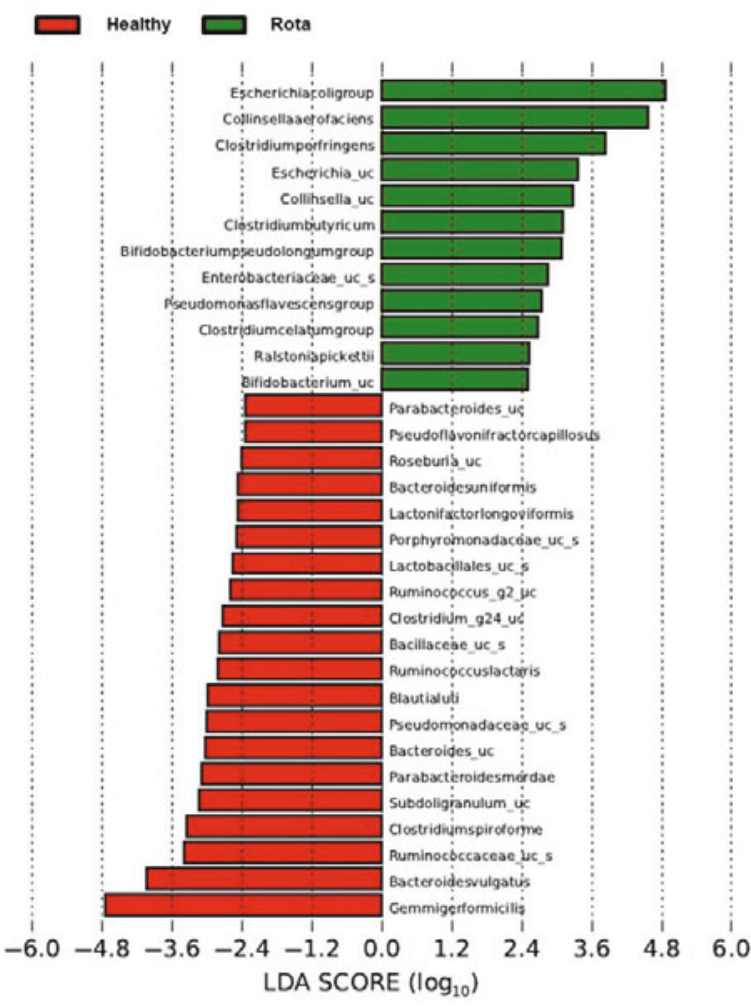

a: Bifidobacterium uc
b: Bificobacteriumpseudolongumgroup
cisicobacterium

c: Bificobacterinm

f: Collinsella Uc

R: Collinselía

Bacteroidesuniformis

Do799557
$:$ Bacteroides

: Bacteroicaceae

Parabacteroides uc

Parabacteroidesmerdae

Porphyromonadace ae uc

5: Porphyromonadace ae ve

t: Porphyromonacaceae

v: clostriciumbutyricum

w: Clostriciumcelitumgroup

a: $A B 06237$ g UC
aO $A B 606237^{\circ} \mathrm{g}$

2. 371547 s

4: Blautialuti

7: DO $804478^{-5}$

a8: EU: 776034

bo: Butyrivibrio 91

2: Clostricium g24_ue

Clostridium $\mathrm{g}^{2}$

EU779209's

$76810005^{5}$

tu 842423 - U

2: 18803959 Uc

4: 11KB $\mathrm{KB}^{-1}$

Lactonifactorlongoviformis

8: Roseburia-

do: Ruminococcuslactaris

2: Ef 401700 s

d6: Go175418.9-uc

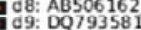

年

e2: N 13389 - 9 s

e4: $A D D X 5$

es: 104528525

seucortavonifractorca pillosus

9: Ruminococcaceae uc

I1: Do057453 s

14: Ruminococcus g2 uc

S: DQ797332 s 92

Gemmigerformicilis

Subdolgranulum_ue

g0: Ruminococcaceae
(C)

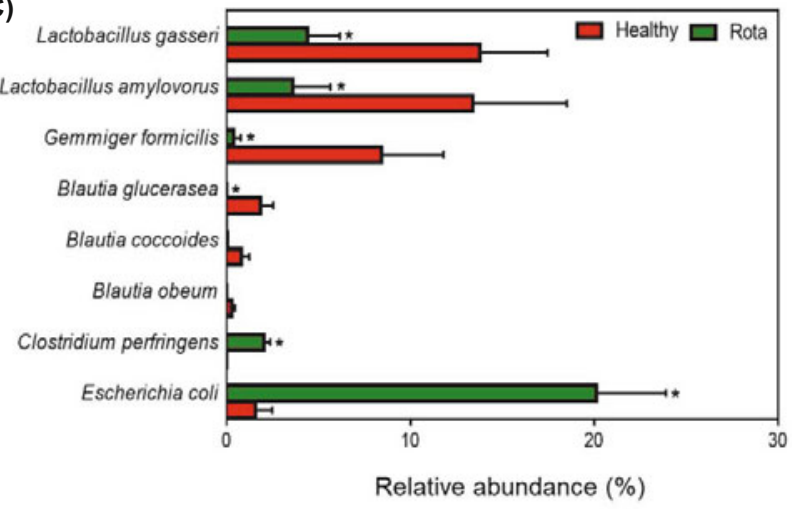

Fig. 5. Distinctive species in the feces of healthy and rotavirus diarrhea calves. (A) Cladogram plotted from LEfSe analysis showing the taxonomic levels represented with phyla in the outermost the ring and species in the innermost ring. Each circle is a member within that level; (B) Plot from LEfSe analysis indicating enriched species associated either with healthy (red) or rotavirus diarrhea (green); (C) Relative abundance of the main bacterial species in feces of healthy and rotavirus diarrhea by Mann-Whitney test. ${ }^{*} P<0.05$. 
Next, we performed a taxonomy composition analysis to identify the species more relevant to rotavirus infection. As a result, 71 and 16 bacterial species were identified in the healthy and rotavirus-infected group, respectively (Fig. 5A). Twenty species including Gemmiger formicillis and Bacteroides vulgatus were shown to be associated with healthy calves, whereas 12 species including E. coli and Clostridium perfringens were significantly associated with rotavirus diarrhea (Fig. 5B). The prevalence of the species that were found to be significant to disease status in this analysis are presented in Fig. 5C. In detail, E. coli and Clostridium perfringens were shown to be significantly increased, while Lactobacillus gasseri, L. amylovorus, Gemmiger formicillis, Blautia coccoides, and B. obeum were significantly decreased in rotavirus diarrhea. These results indicate that many of the top discriminatory core microbiota belong to a few bacterial clades.

\section{Canonical correlation and Pearson correlation coefficient analysis}

To determine whether an altered microbiota structure was correlated with physiological parameters obtained from healthy and rotavirus-infected calves, we performed canonical cor- relation analysis (CCA, Fig. 6). We found that in healthy calves, the genera Lactobacillus, Coprococcus_g2, Bacteroides, Blautia, and Subdoligranulum were shown to be correlated with the serum glucose level. In contrast, the potentially harmful opportunists Clostridium_g21, Clostridium, Streptococcus, and E. coli were correlated with the levels of neutrophils, SAA, BUN, and WBC in diarrheic calves. At the species level, the similarities to serum glucose levels were higher in the order of L. amylovorus, B. glucerasea, G. formicillis, L. gasseri, and $B$. coccoides in healthy calves. However, the diarrheic calves showed a correlation between the relative abundances of $C$. perfringens and E. coli and the levels of WBC, BUN, and SAA.

To evaluate the individual correlations between gut bacteria and physiological parameters, we generated Pearson correlation scores followed by an analysis of variance (Table 1). At the genus level, Lactobacillus, Subdoligranulum, Blautia, and Bacteroides correlated negatively with WBC, BUN, and SAA, whereas they correlated positively with glucose. Importantly, these genera did not correlate with neutrophils. In contrast, Escherichia correlated positively neutrophils and BUN, and Streptococcus correlated positively with WBC, neutrophils, and SAA. At the species level, L. gasseri, G. formicillis, and B. glucerasea correlated negatively with WBC
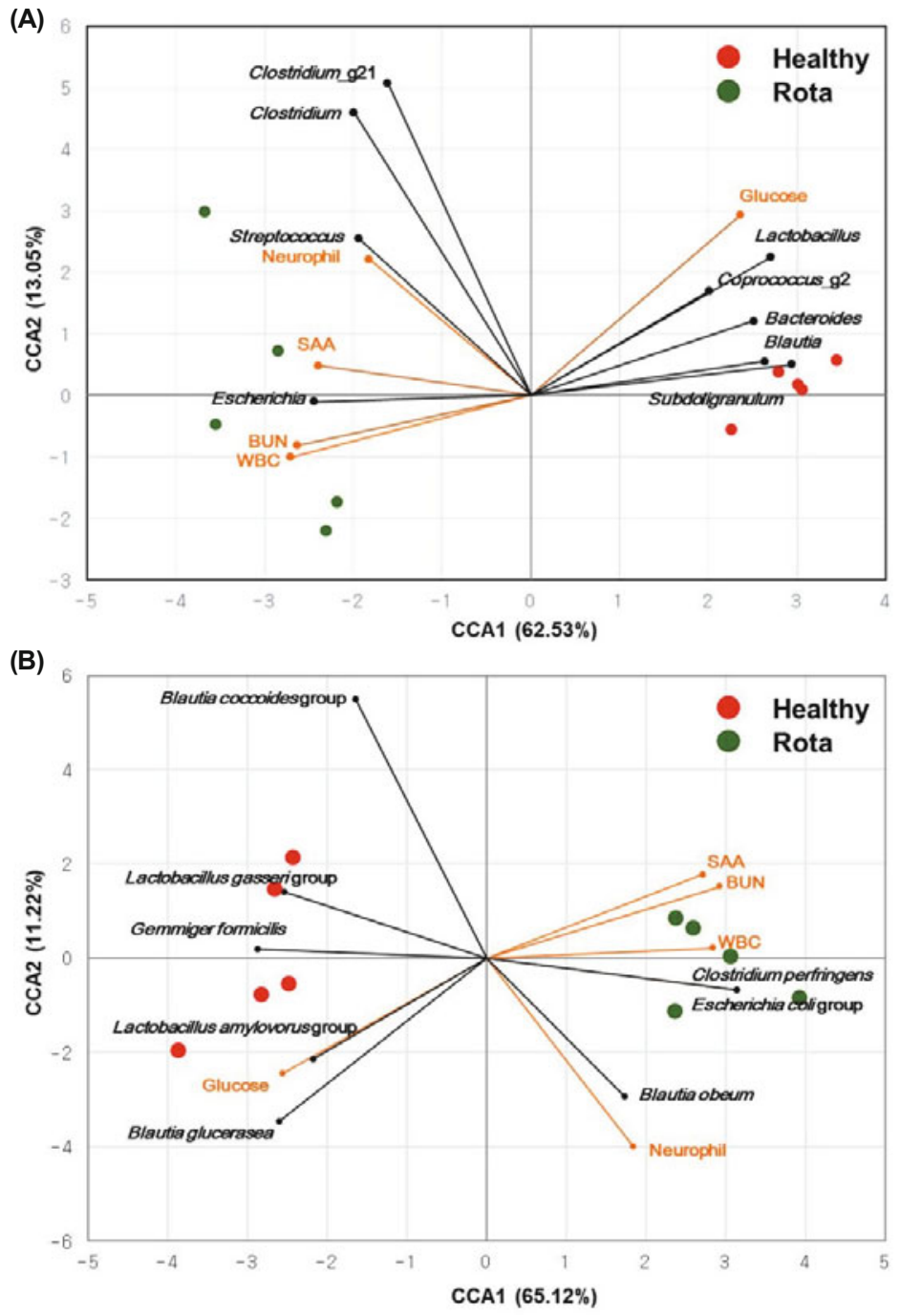

Fig. 6. Canonical correlation analysis (CCA) reveals structural correlations. (A) Genus; (B) Species. Plot of first and second dimension of CCA with labeled healthy (red), rotavirus diarrhea (green), fecal microbiota (black), and physiological parameters (orange). 
Table 1. Pearson correlation coefficient between fecal microbiota and physiological parameters

\begin{tabular}{|c|c|c|c|c|c|}
\hline \multirow{2}{*}{ Fecal microbiota } & \multicolumn{5}{|c|}{ Correlation coefficient $(r)$} \\
\hline & WBC & Neutrophil & Glucose & BUN & SAA \\
\hline \multicolumn{6}{|l|}{ Genus level } \\
\hline Lactobacillus & $-0.80^{* *}$ & -0.32 & $0.83^{* *}$ & $-0.93^{* * *}$ & $-0.76^{*}$ \\
\hline Subdoligranulum & $-0.89^{* * *}$ & -0.55 & $0.78^{\star *}$ & $-0.88^{\star \star \star}$ & $-0.74^{*}$ \\
\hline Blautia & $-0.78^{* *}$ & -0.52 & 0.62 & $-0.71^{\star}$ & -0.56 \\
\hline Bacteroides & $-0.78^{\star *}$ & -0.22 & $0.67^{*}$ & $-0.77^{\star * *}$ & $-0.65^{*}$ \\
\hline Coprococcus_g2 & $-0.66^{*}$ & -0.50 & 0.53 & -0.52 & -0.31 \\
\hline Escherichia & 0.62 & $0.67^{*}$ & -0.60 & $0.72^{*}$ & 0.47 \\
\hline Clostridium_g21 & 0.30 & 0.43 & 0.01 & 0.49 & 0.49 \\
\hline Streptococcus & $0.65^{\star}$ & $0.71^{*}$ & -0.51 & 0.31 & $0.65^{\star}$ \\
\hline Clostridium & 0.49 & 0.50 & -0.16 & 0.58 & 0.60 \\
\hline \multicolumn{6}{|l|}{ Species level } \\
\hline Lactobacillus gasseri & $-0.78^{\star *}$ & -0.33 & 0.46 & $-0.78^{\star *}$ & -0.62 \\
\hline Lactobacillus amylovorus & -0.60 & -0.35 & $0.66^{*}$ & -0.61 & -0.59 \\
\hline Gemmiger formicillis & $-0.78^{* *}$ & -0.60 & $0.76^{*}$ & $-0.87^{\star *}$ & $-0.75^{*}$ \\
\hline Blautia glucerasea & $-0.63^{*}$ & -0.34 & $0.79^{\star *}$ & $-0.88^{* * *}$ & $-0.77^{\star *}$ \\
\hline Blautia coccoides & -0.45 & -0.63 & 0.34 & -0.33 & -0.25 \\
\hline Blautia obeum & 0.30 & 0.43 & 0.01 & 0.49 & 0.49 \\
\hline Clostridium perfringens & $0.87^{\star *}$ & 0.53 & $-0.73^{*}$ & $0.91^{\star * *}$ & $0.84^{* *}$ \\
\hline Escherichia coli & $0.94^{* * *}$ & $0.63^{*}$ & $-0.88^{\star * *}$ & $0.81^{\star *}$ & $0.78^{\star *}$ \\
\hline
\end{tabular}

and BUN, while $C$. perfringens correlated positively with WBC and SAA. Interestingly, E. coli correlated with all clinical characteristics (positively with WBC, neutrophils, BUN, and SAA; negatively with glucose levels). These results indicate a close interrelationship between physiological parameters, gut microbial composition, and rotavirus diarrhea.

\section{Discussion}

A variety of studies have documented the changes in microbiological development in the intestine of calves and in relation to various management practices, such as housing, feeding, and antimicrobial administration during the neonatal period (Lukas et al., 2007; Uyeno et al., 2010; Edrington et al., 2012; Li et al., 2012; Klein-Jobstl et al., 2014). However, there is a limited amount of information regarding the correlation between specific changes in the gut microbiota and the physiological parameters of neonatal calf diarrhea. In this study, we found an altered gut microbiota structure in rotavirus-infection calves and demonstrated a significant association between the gut microbiota and the physiological parameters via feces and serum analysis of healthy and rotavirus diarrheic calves. First, we evaluated the dissimilarity in community membership and structure of the gut microbiota between healthy and rotavirus diarrheic calves. Then, to evaluate the correlation between the gut microbiota and physiological parameters, we generated Pearson correlation scores and performed canonical correlation analyses.

In the present study, a total of $1,221,812$ reads were used for the final analysis, with an average of 81,454 reads per calf. We identified bacterial genera that discriminated between healthy and rotavirus diarrhea. Then, we found low bacterial diversity in rotavirus diarrhea through the analysis of rarefaction curves and alpha-diversity, which is a well-known hallmark of dysbiosis. In the early stages of life in the calves, the incidence of diarrhea was associated with a decrease in bacterial diversity in the gut (Oikonomou et al., 2013).

Less is known about specific gut microbiota which are potentially beneficial to the host. A previous report showed that, at the phylum level, the gut microbiota in healthy calves were dominated by Firmicutes (64-82\%), followed by Bacteroidetes (8-24\%) and Actinobacteria (1-12\%) during the neonatal period (Oikonomou et al., 2013). In this study, we found alterations in the gut microbiota of neonatal diarrhea samples as a result of rotavirus infection. The relative abundances of Firmicutes and Bacteroidetes increased in healthy calves, while Proteobacteria was abundant in rotavirus diarrhea samples, which is consistent with a previous report showing that the phylum Proteobacteria in rotavirus diarrhea is associated with gut dysbiosis and inflammation (Singh et al., 2015). At the genus level, in rotavirus-infected calves, there was significant increase of the genera Escherichia, Clostridium, and Streptococcus. However, Subdoligranulum, Blautia, Bacteroides, and Coprococcus were decreased. Notably, Lactobacillus was significantly decreased in the rotavirus diarrhea group.

Next, we compared the gut microbiota of healthy and rotavirus diarrheic calves at the species level. The profile of the species level was similar to the profile observed in other studies of intestinal dysbiosis. In general, an increased microbial diversity was associated with a healthy intestine, where previous studies associated a reduction of alpha-diversity with infections of $C$. difficile and IBD- and IBS-diarrhea subtypes (Kostic et al., 2014). Our relative abundance analysis revealed that Lactobacillus species such as L. gasseri and $L$. amylovorus were found to be relatively abundant in healthy calves. L. gasseri is known to prevent the severity of acute selflimiting diarrhea in adults, comparable to effective monobacterial medicine (Margreiter et al., 2006). Moreover, the re- 
lative abundance of G. formicillis, B. glucerasea, B. coccoides, and $B$. obeum were significantly higher in the healthy group. In contrast, the study identified two species of $C$. perfringens and E. coli that were associated with the diarrheic calves.

Our Pearson correlation results showed that Lactobacillus, Subdoligranulum, Blautia, and Bacteroides were negatively correlated with WBC, BUN, and SAA levels, but positively correlated with glucose level. In contrast, Escherichia and Streptococcus positively correlated with physiological parameters, notably neutrophil levels. At the species level, L. gasseri, G. formicillis, and B. glucerasea were negatively correlated with WBC, BUN, and SAA levels. Notably, the prevalence of the E. coli was associated with all physiological parameters (positively WBC, neutrophils, BUN and SAA, and negatively glucose), and the incidence of rotavirus diarrhea. Interestingly, the E. coli was the only species that positively correlated with neutrophils.

The optimization of feed efficiency and diarrhea prevention without antibiotics is a major goal of any livestock production system. In this regard, our results that rotavirus infection alters the structure of the gut microbiota, which correlates changes in physiological parameters would provide new insight into the treatment of rotavirus-mediated diarrhea in calves.

\section{Acknowledgements}

This work was supported by the Ministry of Education of the Republic of Korea and the National Research Foundation of Korea (NRF-2017R1A2B2005685) and partially supported by a grant from the World Institute of Kimchi (KE1802-1), funded by the Ministry of Science and ICT, Republic of Korea. The authors are grateful to the graduate students of Chonbuk National University, Gyeongsang National University, and Kyungpook National University who supported sample collection and analysis. We would also like to thank Kyoung-Seong Choi, Hyeon-Cheol Kim, and Bae-Keun Park for their assistance in detecting pathogens.

\section{Conflict of Interest}

The authors declare that they have no competing interests.

\section{References}

Ammar, S.S., Mokhtaria, K., Tahar, B.B., Amar, A.A., Redha, B.A., Yuva, B., Mohamed, H.S., Abdellatif, N., and Laid, B. 2014. Prevalence of rotavirus (GARV) and coronavirus (BCoV) associated with neonatal diarrhea in calves in western Algeria. Asian Pac. J. Trop. Biomed. 4, S318-S322.

Cho, Y.I., Kim, W.I., Liu, S., Kinyon, J.M., and Yoon, K.J. 2010. Development of a panel of multiplex real-time polymerase chain reaction assays for simultaneous detection of major agents causing calf diarrhea in feces. J. Vet. Diagn. Invest. 22, 509-517.

Cho, Y.I. and Yoon, K.J. 2014. An overview of calf diarrhea - infectious etiology, diagnosis, and intervention. J. Vet. Sci. 15, 1-17.

Edrington, T.S., Dowd, S.E., Farrow, R.F., Hagevoort, G.R., Callaway, T.R., Anderson, R.C., and Nisbet, D.J. 2012. Development of colonic microflora as assessed by pyrosequencing in dairy calves fed waste milk. J. Dairy Sci. 95, 4519-4525.

Gomez, D.E., Arroyo, L.G., Costa, M.C., Viel, L., and Weese, J.S. 2017. Characterization of the fecal bacterial microbiota of healthy and diarrheic dairy calves. J. Vet. Intern. Med. 31, 928-939.

Gulliksen, S.M., Jor, E., Lie, K.I., Hamnes, I.S., Loken, T., Akerstedt, J., and Osteras, O. 2009. Enteropathogens and risk factors for diarrhea in Norwegian dairy calves. J. Dairy Sci. 92, 5057-5066.

Hur, T.Y., Jung, Y.H., Choe, C.Y., Cho, Y.I., Kang, S.J., Lee, H.J., Ki, K.S., Baek, K.S., and Suh, G.H. 2013. The dairy calf mortality: the causes of calf death during ten years at a large dairy farm in Korea. Korean J. Vet. Res. 53, 103-108.

Izzo, M.M., Kirkland, P.D., Mohler, V.L., Perkins, N.R., Gunn, A.A., and House, J.K. 2011. Prevalence of major enteric pathogens in Australian dairy calves with diarrhoea. Aust. Vet. J. 89, 167-173.

Kapikian, A.Z. and Chanock, R.M. 1996. Rotaviruses, pp. 1657-1708. In Straus, S.E. (ed.), Fields Virology Vol. 2. Lippincott-Raven, Philadelphia, USA.

Klein-Jobstl, D., Schornsteiner, E., Mann, E., Wagner, M., Drillich, M., and Schmitz-Esser, S. 2014. Pyrosequencing reveals diverse fecal microbiota in simmental calves during early development. Front. Microbiol. 5, 622.

Kostic, A.D., Xavier, R.J., and Gevers, D. 2014. The microbiome in inflammatory bowel disease: current status and the future ahead. Gastroenterology 146, 1489-1499.

Li, R.W., Connor, E.E., Li, C., Baldwin Vi, R.L., and Sparks, M.E. 2012. Characterization of the rumen microbiota of pre-ruminant calves using metagenomic tools. Environ. Microbiol. 14, 129-139.

Lukas, F., Koppova, I., Kudrna, V., and Kopecny, J. 2007. Postnatal development of bacterial population in the gastrointestinal tract of calves. Folia Microbiol. 52, 99-104.

Margreiter, M., Ludl, K., Phleps, W., and Kaehler, S.T. 2006. Therapeutic value of a Lactobacillus gasseri and Bifidobacterium longum fixed bacterium combination in acute diarrhea: a randomized, double-blind, controlled clinical trial. Int. J. Clin. Pharmacol. Ther. 44, 207-215.

Millar, H.R., Simpson, J.G., and Stalker, A.L. 1971. An evaluation of the heat precipitation method for plasma fibrinogen estimation. J. Clin. Pathol. 24, 827-830.

Oikonomou, G., Teixeira, A.G., Foditsch, C., Bicalho, M.L., Machado, V.S., and Bicalho, R.C. 2013. Fecal microbial diversity in preweaned dairy calves as described by pyrosequencing of metagenomic $16 \mathrm{~S}$ rDNA. Associations of Faecalibacterium species with health and growth. PLoS One 8, e63157.

Penders, J., Thijs, C., Vink, C., Stelma, F.F., Snijders, B., Kummeling, I., van den Brandt, P.A., and Stobberingh, E.E. 2006. Factors influencing the composition of the intestinal microbiota in early infancy. Pediatrics 118, 511-521.

Rolhion, N. and Chassaing, B. 2016. When pathogenic bacteria meet the intestinal microbiota. Philos. Trans. R. Soc. Lond. B Biol. Sci. 371, 20150504.

Singh, P., Teal, T.K., Marsh, T.L., Tiedje, J.M., Mosci, R., Jernigan, K., Zell, A., Newton, D.W., Salimnia, H., Lephart, P., et al. 2015. Intestinal microbial communities associated with acute enteric infections and disease recovery. Microbiome 3, 45.

Uetake, K. 2013. Newborn calf welfare: a review focusing on mortality rates. Animal Sci. J. 84, 101-105.

USDA. 2008. Dairy 2007 Part II: changes in the US dairy cattle industry, 1991-2007. Fort Collins: USDA-APHIS-VS, CEAH, 57-61.

Uyeno, Y., Sekiguchi, Y., and Kamagata, Y. 2010. rRNA-based analysis to monitor succession of faecal bacterial communities in Holstein calves. Lett. Appl. Microbiol. 51, 570-577.

Wotzka, S.Y., Nguyen, B.D., and Hardt, W.D. 2017. Salmonella Typhimurium diarrhea reveals basic principles of enteropathogen infection and disease-promoted DNA exchange. Cell Host Microbe 21, 443-454. 